\title{
HISTIOCITOMA MALIGNO FIBROSO RETROPERITONEAL
}

\author{
E. ARGÜELLES SALIDO, C.B. CONGREGADO RUIZ, R.A. MEDINA LÓPEZ, \\ J.L. PASCUAL DEL POBIL MORENO
}

Servicio de Urología. Unidad de Uro-Oncología. Hospital Universitario Virgen del Rocío. Sevilla.

Actas Urol Esp. 28 (8): 624-626, 2004

\section{RESUMEN}

HISTIOCITOMA MALIGNO FIBROSO RETROPERITONEAL

Presentamos un nuevo caso de neoplasia retroperitoneal: un histiocitoma fibroso maligno, en su variedad mixoide, la menos agresiva de ellas. Este es un tumor individualizado de forma relativamente reciente del conjunto de sarcomas retroperitoneales, de escasa frecuencia, clínica inespecífica y mal pronóstico por su alta tendencia a la recidiva y a la producción de metástasis.

PALABRAS CLAVE: Retroperitoneo. Histiocitoma fibroso maligno. Tumor.

\section{ABSTRACT}

\section{RETROPERITONEAL MALIGNANT FIBROUS HISTIOCYTOMA}

We report a new case of retroperitoneal tumor: a malignant fibrous histiocytoma, in its less agressive histologyc type, the mixoyd variety. This is a recently isolated histologyc presentation from other sarcomas, with a low incidence, non-specific clinic findings and poor prognosis due to its tendency to reappear and to produce metastasis.

KEY WORDS: Retroperitoneum. Malignant fibrous histiocytoma. Tumor.

$\mathrm{L}$ os tumores retroperitoneales son neoformaciones benignas o malignas originadas en los distintos elementos que conforman este espacio, excluyendo aquellos procedentes de órganos situados en él. En un 85\% de las ocasiones aparecen como malignos, correspondiendo un 35\% de ellos a sarcomas.

El objetivo de este trabajo es exponer un nuevo caso de histiocitoma fibroso maligno de tipo mixoide de localización retroperitoneal pararrenal, dada su infrecuente presentación.

\section{CASO CLÍNICO}

Varón de 70 años, sin antecedentes de interés, que consultó por síndrome constitucional de 5 meses de evolución asociado a polaquiuria.

Exploración física sin alteraciones. Analíticamente sólo destacaba una cifra de hemoglobina de 9,17 gr/dL, con un hematocrito de 0,31, además de una trombocitosis de 733.000 por microlitro.
La ecografía puso de manifiesto una gran masa sólida, heterogénea, con imágenes sospechosas de necrosis en su interior, situada entre bazo y riñón izquierdo.

La tomografía axial computerizada (TAC) confirmaba la presencia de una neoformación de aproximadamente $10 \times 10 \mathrm{~cm}$, heterogénea y que captaba contraste, presentando zonas hipodensas en su interior. Se situaba inmediatamente por detrás del riñón izquierdo al que desplazaba hacia delante, dando la impresión de depender de la cortical de éste (Fig. 1).

Ante estos hallazgos se decidió tratamiento quirúrgico, encontrándose una masa retroperitoneal de la que participaba el riñón, por lo que se procedió a la exéresis de ambos. No hubo incidencias destacables en el post-operatorio.

La anatomía patológica mostró un riñón con presencia de quistes corticales y leves lesiones de pielonefritis. La masa se evidenció como indepen- 


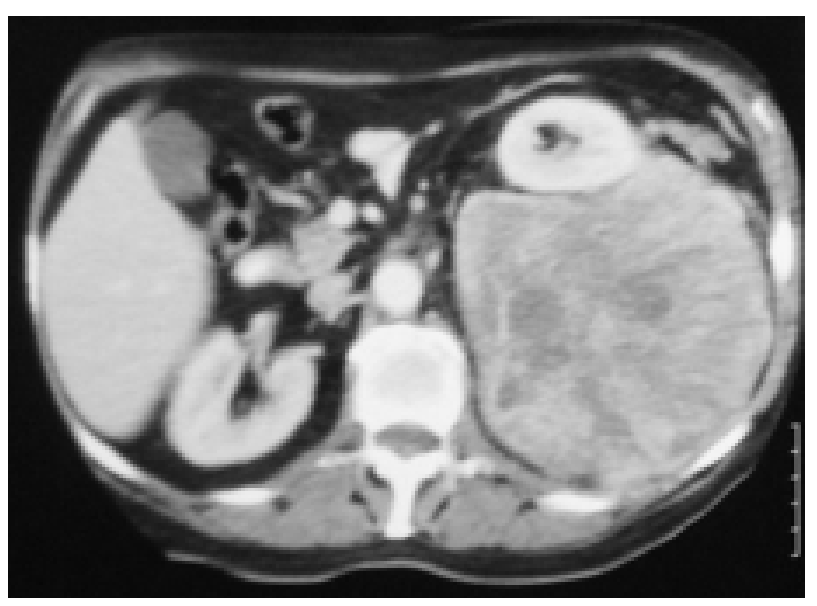

FIGURA 1. Gran masa sólida heterogénea situada por detrás del riñón izquierdo.

diente del riñón siendo etiquetada como histiocitoma fibroso maligno retroperitoneal de tipo mixoide. Los estudios inmunohistoquímicos dieron positividad para CD68 y $\alpha 1$ quimiotripsina, pero con Desmina y Myo4 negativos (Fig. 2).

Tras aproximadamente 21 meses, el paciente desarrolló metástasis localizadas en trígono retromolar y sobre ambos campos pulmonares, siendo sometido a tratamiento quimioterápico con ifosfamida y adriamicina. Secundariamente al tratamiento desarrolla una neutropenia febril que obliga a su ingreso dada su mala situación general. El cuadro es tratado con amikacina y cefepina, siendo superado por el paciente.

Actualmente, 24 meses después de la intervención continúa sus revisiones por el Servicio de Oncología Médica.

\section{DISCUSIÓN}

El histiocitoma fibroso maligno, de identificación relativamente reciente, es la forma de sarcoma más frecuente de la edad adulta, pudiendo afectar a cualquier grupo de edad, y siendo muy infrecuente en la infancia ${ }^{1}$. La serie presentada por Weiss en 1978 con doscientos casos continúa siendo una de las más importantes aportadas hasta ahora ${ }^{2}$.

$\mathrm{Su}$ frecuencia oscila entre el 0,2 y el 0,6\% del total de tumores ${ }^{3}$.

Aparece por lo general en tejidos blandos, sobre todo en las extremidades, con sólo un 12$15 \%$ de casos de aparición en el retroperitoneo ${ }^{4}$.

Su histogénesis se ha mostrado controvertida aunque la hipótesis más admitida es que su origen se encuentra en células mesenquimales pluripotentes, lo que explica que a veces pueda coincidir con formaciones como el lipo o el fibrosarcoma, e incluso con enfermedades hematológicas ${ }^{1,5,6}$.

Histológicamente se han descrito diversas variantes: fibroso de células gigantes, inflamatorio y mixoide, siendo la última la más frecuente ${ }^{3}$, en la que se encuentran grandes áreas hipercelulares, con células en forma de huso o estrella y que se agrupan en un patrón fascicular en un tejido mixoide muy rico en ácido mucopolisacárido. Desde un punto de vista inmunohistológico estos tumores expresan positividad a la quimotripsina y al CD 68 . La variedad mixoide, como el caso que nos ocupa, es la que parece demostrar un comportamiento menos agresivo si la comparamos con las demás ${ }^{4}$.

Su clínica, al igual que la de otros tumores retroperitoneales, aparece como inespecífica, derivando del tamaño alcanzado o de su carácter de malignidad, que le lleva a hacer participar estructuras y órganos vecinos ${ }^{7}$. Su síntoma predominante es el dolor del flanco afecto. Se presenta también como síndrome constitucional con anorexia, astenia y pérdida de peso $^{8}$, forma con la que debutó el caso que nos ocupa.

Analíticamente puede observarse de manera ocasional leucocitosis, anemia y/o elevación de productos ureicos nitrogenados (BUN). La forma inflamatoria suele producir fiebre y leucocitosis con eosinofilia y neutrofilia, que se resuelve con la extirpación tumoral. Este fenómeno se supone debido a la presencia de factores mielopoyéticos ${ }^{9}$.

Macroscópicamente las lesiones son grandes, grisáceas, multinodulares, con áreas secundarias de hemorragia y necrosis, como otros sarcomas de alto grado. Actualmente la creencia sobre su origen es que prácticamente la totalidad de ellos crecen fuera del parénquima renal, aunque un pequeño número lo puede hacer de la cápsula o de elementos tisulares procedentes del hilio ${ }^{9}$.

En cuanto a su diagnóstico las pruebas radiográficas simples en ocasiones muestran calcificaciones (10\% de los casos) con distribución focal o difusa. La realización de urografía intravenosa (UIV) puede demostrar desplazamiento del propio riñón, los uréteres o incluso la vejiga. Ecográficamente el patrón que presentan estos tumores es comúnmente hipoecoico con algunos ecos en su interior, aunque ocasionalmente aparecen como patrón mixto, y rara vez quístico. En la TAC 


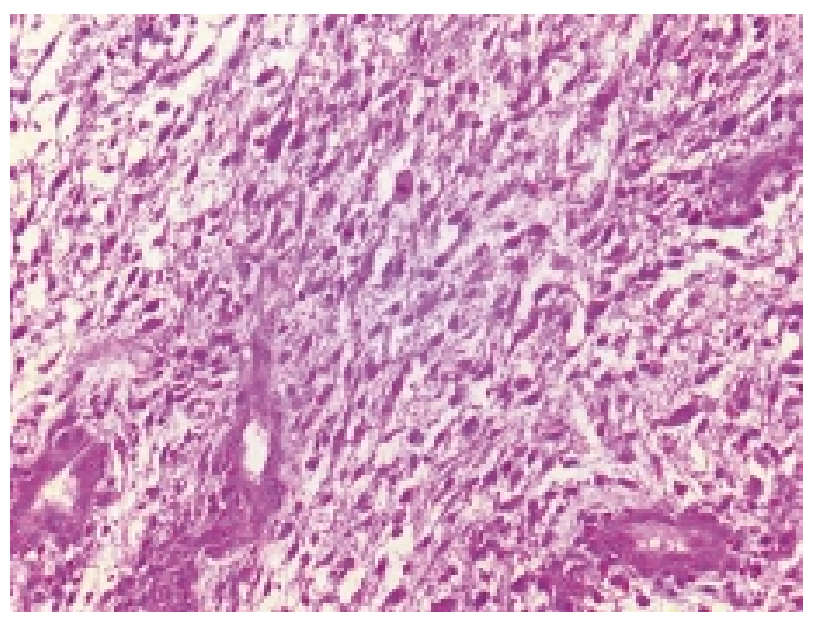

FIGURA 2. Inmunohistoquimica: positividad para CD68 y $\alpha 1$ quimiotripsina.

usualmente se evidencian con aspecto sólido, con algunas áreas de baja densidad, que se corresponden con zonas de necrosis o hemorragia ${ }^{9}$. Esta prueba ayuda a delimitar bien el tamaño y localización del tumor, lo que a su vez permitirá un mejor planteamiento del abordaje quirúrgico (a lo que puede contribuir la angiografia), y seguimiento postoperatorio. Que duda cabe que esta exploración ha sufrido un gran impulso con el desarrollo de la tecnología helicoidal y programas que van a permitir una reconstrucción tridimensional o de paletas de color basadas en cálculo de los coeficientes de atenuación lineal de los distintos tejidos. La resonancia magnética nuclear (RMN) ha supuesto una gran progresión en relación con el comportamiento de los distintos tejidos bajo la acción de pulsos secuenciados de radiofrecuencias.

Como en todos los tumores retroperitoneales, el tratamiento quirúrgico continúa siendo la única terapia con posibilidades curativas, aunque para ello es necesaria la resección completa de la neoformación. Esto conlleva en ocasiones la extirpación de órganos adyacentes para asegurar la radicalidad del gesto quirúrgico. Su vía de abordaje vendrá condicionada fundamentalmente por el tamaño del tumor, localización y posible infiltración de órganos vecinos.

La tasa de recurrencia de los tumores retroperitoneales es alta, entre un 50-82\%, dependiendo particularmente de su naturaleza, del tamaño tumoral, grado y estadio ${ }^{1}$. Por ello el pronóstico suele ser malo, con tendencia a metastatizar en pulmón, hígado, hueso y médula ósea ${ }^{1,5}$.
El papel de la radioterapia y quimioterapia en el tratamiento de estas neoplasias retroperitoneales sigue siendo motivo de opiniones controvertidas aunque no parecen aumentar la supervivencia global ${ }^{10}$. El hecho de un posible aumento del periodo libre de enfermedad hace que sea propugnada en la mayoría de los casos ante las dificultades que entraña la exéresis completa ${ }^{1}$.

Se ha ensayado también la utilización de quimiofármacos, bien en monoterapia o en combinación de varios, no habiéndose encontrado aún el régimen más idóneo. La adriamicina se ofrece como droga de base bien aislada o asociada con el DIC (dimetil-triaceno-imidazol-carbosmida) y vincristina ${ }^{5}$.

\section{REFERENCIAS}

1. Zuluaga Gómez A, Torres Ramírez C, García Gil JM et al. Tumores retroperitoneales primitivos de origen histiocitario. Act Urol Esp 1984;4:279.

2. Weiss SW, Enzinger FM. Malignant fibrous histiocytoma: an analysis of 200 cases. Cancer 1978;41:2250-2566.

3. García Freire C, Villar M, López Pita J, Martínez S. Histiocitoma fibroso benigno retroperitoneal. Presentación de un caso. Actas Urol Esp 1998;22(6):524-527.

4. Virgili G, Di Stasi SM, Storti L, Orlandi A, Vespasiani G. Successful management of retroperitoneal malignant fibrous histiocytoma involving both kidneys. Scand J Urol Nephrol 2000;34:208-210.

5. Garmendia JC, Arocena F, Sanza JP et al. Histiocitoma fibroso maligno retroperitoneal tipo inflamatorio: a propósito de un caso. Arch Esp Urol 1989;42:922-925.

6. Zuluaga Gómez A: Urología y retroperitoneo. Tema monográfico LXII Congreso Nacional de Urología. Valladolid, 1997.

7. Herrero JA, Páez A, Aramburu J et al. Histiocitoma fibroso maligno retroperitoneal tipo mixoide con infiltración de mesocolon. Arch Esp Urol 1995;47:722-725.

8. Del Rosario J, Artiles J, Rodríguez N et al. Histiocitoma fibroso maligno retroperitoneal. Un nuevo caso. Arch Esp Urol 1983;36:213.

9. Goldman SM, Hartman DS, Weiss SW. The varied radiographic manifestations of retroperitoneal malignant fibrous histiocytoma revealed through 27 cases. J Urol 1986;135(1):33-38.

10. Shiloni E, Szold A, White DE, Freund HR. High-grade retroperitoneal sarcomas role of an aggressive palliative approach. J Surg Onc 1993;53:197.

Dr. E. Argüelles Salido

Servicio de Urología. Unidad de Uro-Oncología

Hospital Univ. Virgen del Rocío

Avda. Manuel Siurot, s/n - 41013 Sevilla

earguelles@telefonica.net

(Trabajo recibido el 2 septiembre de 2003) 